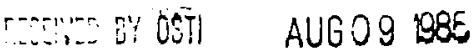

UCRL--92172

DE85 016071

\section{ELECTROMAGIIETIC EFFECTS II RELATIVISTIC ELECTRON BEAM PLASMA IIITERACTIOIIS}

\author{
H. L. Kruer \\ A. B. Langdon
}

This paper was prepared for the Proceedinas of the Second lorksion on Laser Acceleration of Particles held in Los Anneles, California, Januar. $7-18,1905$.

February 13, 1985

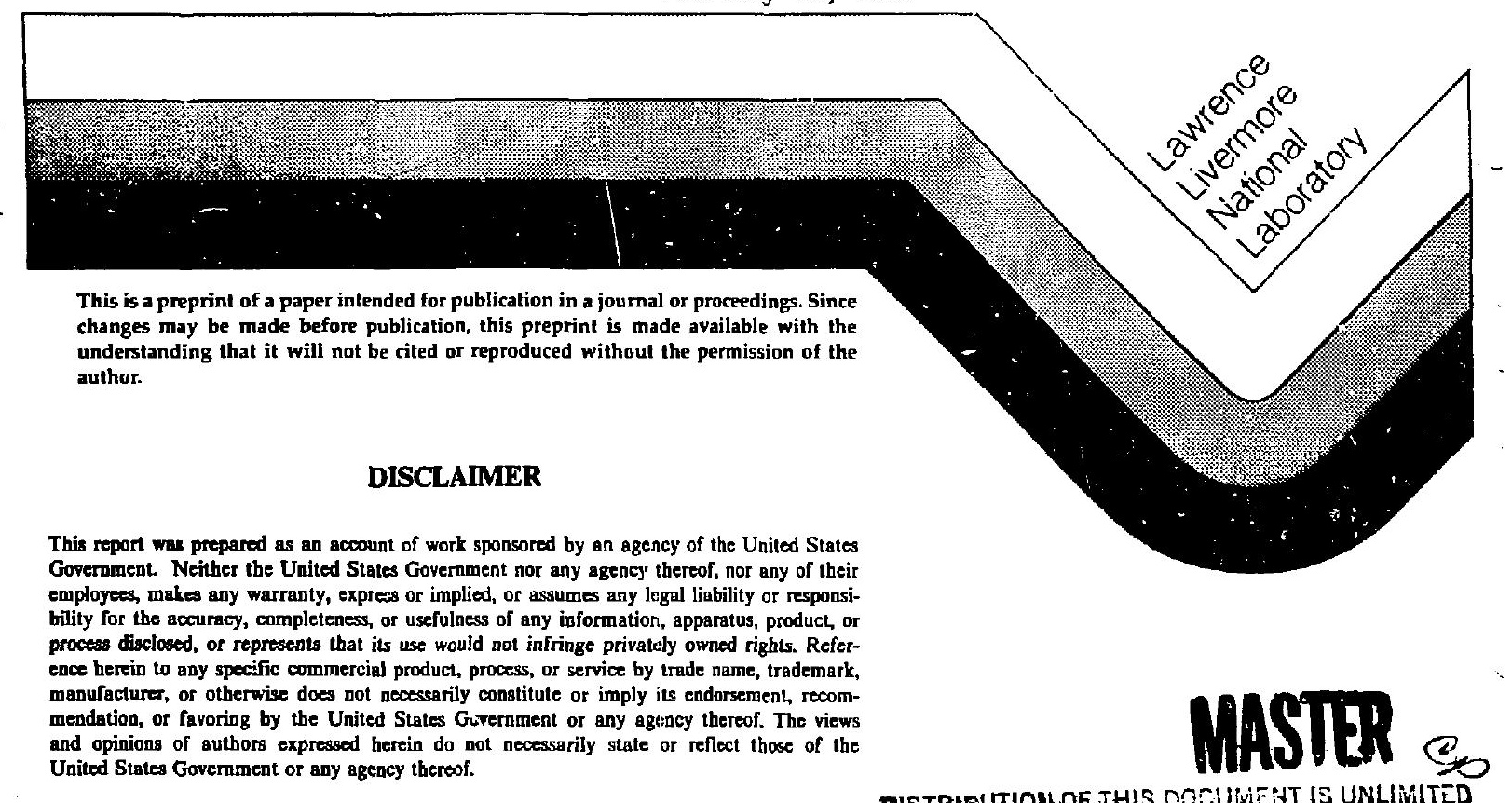




\title{
ELECTROMAGNETIC EFFECTS IN RELATIVISTIC ELECTRON BEAM PLASMA INTERACTIONS
}

\author{
W. L. Kruer and A. B. Langdon \\ Physics Department \\ Lawrence Livermore National Laboratory \\ Livermore, CA 94550
}

\begin{abstract}
Electromagnetic effects excited by intense relativistic electron beams in plasmas are investigated using a two-dimensional particle code. The simulations with dense beams show large magnetic fields excited by the Weibel instability as well as sizeable electromagnetic radiation over a significant range of frequencies. The possible relevance of beam plasma instabilities to the laser acceleration of particles is briefly discussed.
\end{abstract}

\section{INTRODUCTION}

Beam-plasma instabilities are of interest in many applications. These include plasma heating, space and astrophysical plasmas, the generation of radiation, and perhaps even some laser particle accelerators. There's been a great deall of previous theory and computer simulation of beam-plasma instabilities, primarily motivated by applications to plasma heating. Our work is motivated by some recent experiments ${ }^{2}$ by Kato, Benford and Tzach. In these experiments, the electromagnetic radiation due to a relativistic electron beam interacting with a plasma was measured. When the beam density was comparable to the plasma density, it was found that a significant fraction of the beam energy was converted to microwaves (of order $0.2 \%)$. These waves had a broad range of frequencies, extending to many times the electron plasma frequency wpe, rather than simply having frequencies of wpe and its harmonics.

To investigate the beam-plasma interaction, we have used a two-dimensional electromagnetic, relativistic particle code. ${ }^{3}$ The beam travels in the $x$-direction, and the evolution of $E_{x}$, $E_{y}$ and $B_{z}$ is followed in the $x-y$ plane. We started with the simplest problem-following the temporal evolution of an electron beam in a uniform plasma with no guide magnetic field. The boundary conditions were doubly-periodic, and the simulation bcx was rectangular with $\mathrm{L}_{-x}=10.9 \mathrm{c} / \omega_{\mathrm{pe}}$ and $\mathrm{L}_{y}=50.2$

$c / w_{p e}$, where $c$ is the veiocity of light and wpe is the electron plasma frequency. The results changed by less than about $20 \%$ when both $L_{x}$ and $L_{y}$ were halved. The ions were

FWork performed under the auspices of the U. S. Department of Energy by the Lawrence Livermore National Laboratory urider contract number $W-7405-E N G-48$. 
usually treated as a fixed neutralizing background, and 800,000 electrons were used. Initially the beam had a kinetic energy of approximately $0.5 \mathrm{Mev}$ (i.e., $Y=2$ ) with a very narrow themal spread of $50 \mathrm{eV}$. The background electrons had a temperature of $2.5 \mathrm{keV}$ and were initialized with a drift in order to balance the initial beam current. The beam density $\left(n_{b}\right)$ ranged from 0.05 to 0.5 of the total electron density $\left(n_{t}\right)$.

The beam-plasma interaction in these simulations is dominated by two instabilities. The first is the well-known electronelectron beam instability, in which electrostatic waves are driven unstable. For a beam with a narrow spread in energy and angle and for $n_{b} / n_{p} \gamma_{b}=1 / 20$, the results for the frequency and growth rate of the most unstable mode are

$$
\begin{aligned}
& \frac{\omega}{\omega_{p e}}=1-1 / 2\left(\frac{n_{b}}{2 n_{p} \gamma_{b}}\right)^{1 / 3} f(\theta) \\
& \frac{r}{\omega_{p e}}=\sqrt{3 / 2}\left(\frac{n_{b}}{2 n_{p} \gamma_{b}}\right)^{1 / 3} f(\theta),
\end{aligned}
$$

where $f(\theta)=\left(\sin ^{2} \theta+\frac{\cos ^{2} \theta}{\gamma_{b}^{2}}\right)^{1 / 3}$.

Here $n_{b}\left(\gamma_{b}\right)$ is the density (gamma) of the beam, $n_{p}$ the plasma density, and $v_{b}$ is the beam velocity. In addition, $\theta$ is the angle between the wave vector $k$ and $v_{b}$, and $k-v_{b}=$ $\omega_{p e}$. The other instability corresponds to $\bar{a}$ purely growing, spatially varying magnetic field transverse to the beam. The maximum growth rate occurs for $\theta=90^{\circ}$ and is

$$
\frac{\gamma}{\omega_{p e}}=\left(\frac{n_{b}}{\left.\gamma_{b}^{n}\right)^{1 / 2}} \frac{k v_{b}}{\left(k^{2} c^{2}+\omega_{p e}\right)^{2}}\right. \text {, }
$$

This Heibel instability occurs since parallel currents attract. Hence beam electrons tend to bunch together or coalesce into filaments, as do also the background electrons carrying the return current. Of course, this instability also produces an electrostatic field which expells background electrons from a filament in order to preserve quasi-neutralitiy. 
To illustrate the simulation results, let's consider a robust example in which $n_{b}=n_{P}$. The electrostatic field energy (EL) versus time is shown in Fig. 1 .

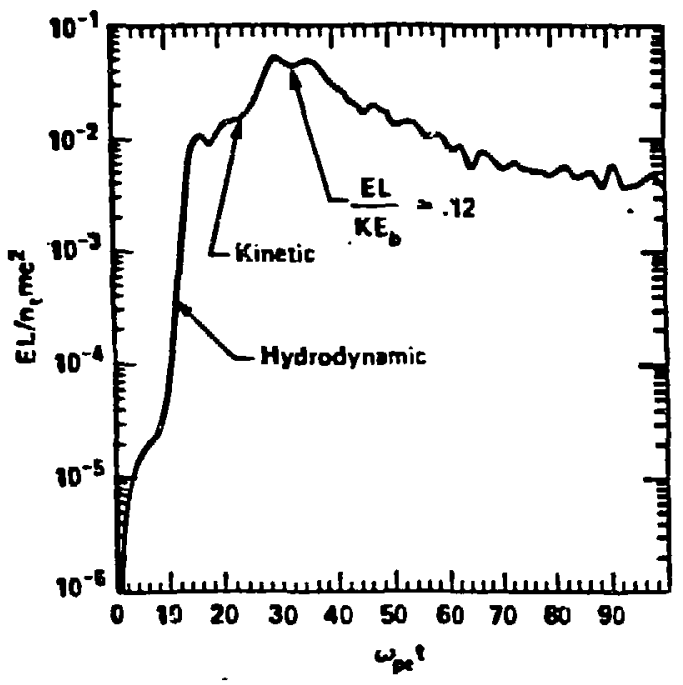

Fig. 1. Evolution of the electrostatic field energy in a 2-D simulation $\left(\gamma_{b}=2, n_{b}=n_{p}\right)$.

Note that early in time there is very rapid growth $(\gamma=1 / 2$ $\left.\omega_{\text {pe }}\right)$, followed by a period of slower growth as the beam nonlinearly develops a significant spread in energy and angles. Finally, the wave energy saturates at about $12 \%$ of the initial kinetic energy of the beam as the velocity distribution becomes monotonic. The wave energy thereafter slowly decays.

The magnetic field energy $\left(B \frac{2}{2} / A_{m}\right)$ versus time is

shown in Fig. 2. The magnetic field due to the Weibel instability initially grows very rapidly $\left(\gamma=1 / 2 \omega_{p e}\right)$, and then grows more slowly as the beam spreads in energy and angles. Finally the magnetic field saturates at a quite large value which corresponds to $\omega_{c e} / \omega_{p e}=0.5$, where $\omega_{c e}=\mathrm{eB} / \mathrm{mc}$ is the electron cyclotron frequency for a nonrelativistic particle.

As the unstable waves grow and saturate, electromagnetic waves are generated as shown in Fig. 3. In this example, the transverse electric field energy (EM) reaches about $2 \%$ of the kinetic energy of the beam $\left(K_{\mathrm{b}}\right)$. These electromagnet ic waves have a significant range of frequencies, rather than just a 


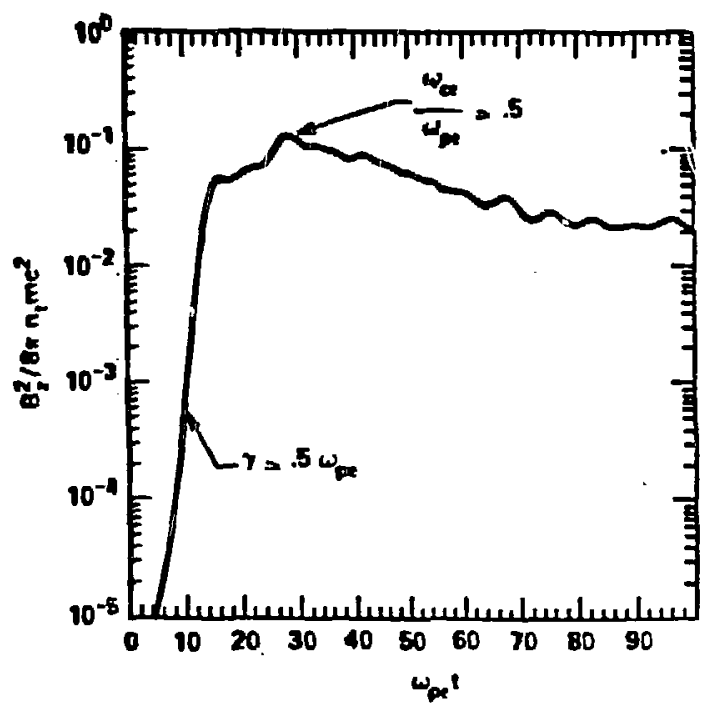

Fig. 2. Evolution of the magnetic field energy in a 2-D simulation.

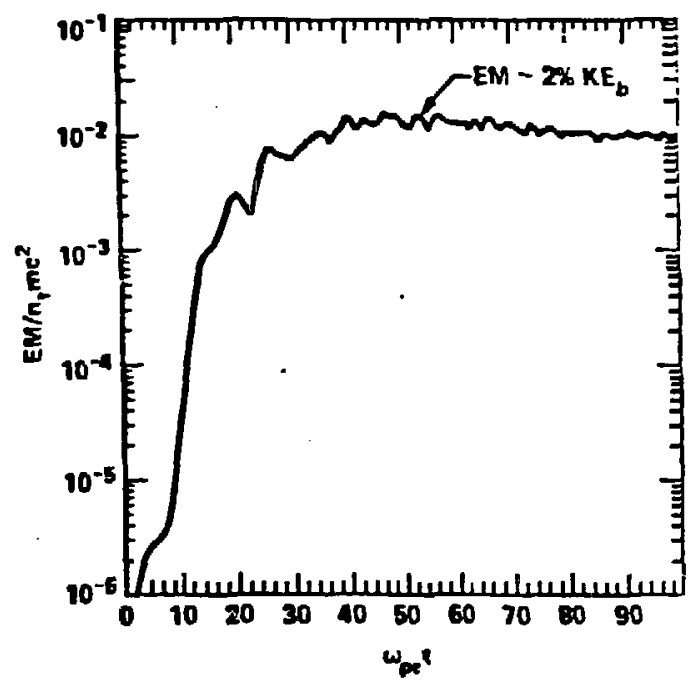

Fig. 3. Evolution of the transverse electric field energy (EM) in a 2-D simulation. 
frequency of wpe and its harmonics. Figure 4 shows the Fourier transform of $B_{2}$ at a given location in the plasma.

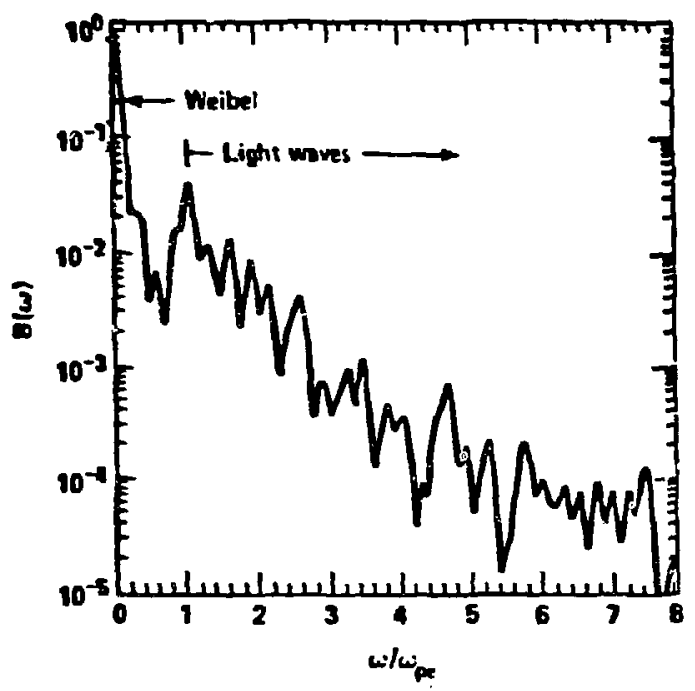

Fig. 4. Fourier transform of the magnetic field at a specific location in the plasma $\left(\gamma_{b}=2, n_{b}=n_{b}\right)$

Note the large peak at $\omega=0$, which is due to the Weibel instability, followed by a signal extending from whe to 3-4 wpe, which is due to light waves. A broad range of frequencies is generated, a feature noted in the experiments. However, the amplitude of the light waves falls off more rapidly with frequency than experimentaliy observed.

Weaker beam densities have aiso been simulated. For a $\gamma_{b}=2$ beam with $n_{b} / n_{t}=0.3$, the peak magnetic field is $\omega_{\mathrm{ce}} / \omega_{\mathrm{pe}}=0.17$, and about $0.3 \%$ of the kinetic energy of the beam is converted to transverse electric fields. For $n_{b} / n_{t}=0.05, \omega_{c e} / \omega_{p e}=0.017$ and the fraction into transverse electric fields is only about $0.06 \%$. In all these cases, the beam loses about $40 \%$ of its energy to the plasma. The beam ends up with a spread in velocity comparable to its initial velocity as well as a broad range of angles.

Although we obtain many of the qualitative features observed in experiments, our simulations are very idealized. The transient temporal problem does not do justice to effects such as those associated with ion motion. For example, noice in Fig. I that the electrostatic field rises and decays in a time of $\approx 100$

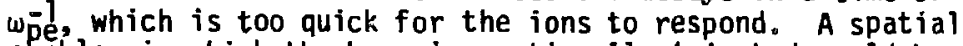
problem in which the beam is continually injected would be more realistic and would also admit boundary conditions closer to 
those in experiments. Allowance for spatial variations in the background plasma density and beam quality may also be important for details of the radiation generation and the power spectrum. Finally our simulations indicate that beam plasma interactions (electrostatic and electromagnet jc) might play a role in schemes such as the beat wave accelerator. ${ }^{4}$ The effect of these instabilitius will depend on factors such as the beam density, energy. and spatiąl width and structure. In experiments currently planned at UCLA, 5 it is estimated that $n_{b} / n_{p}=$ $10^{-2}$ and $\gamma_{p}=20$. The growth rate of the

electron-electron beam instability is then $\simeq 0.05 \mathrm{wpe}$, and

that of the Weibel instability is $=0.02$ wpe. For future applications, the beam density (and $\gamma_{b}$ ) wilf be significantly larger. However, it is not clear that these instabilities will have a deleterious effect. The finite radial extent of the beam can be a stabilizing influence, limiting the wave vectors of the unstable waves. In addition, the finite extent of the beam bunch may allow the beam to "outrun" the unstable fluctuations, 6 particularly since they have wave vectors at significant angles to the beam. Improved simulations can help clarify potential limitations as well as guide experiments.

\section{ACKNOWLEDGMENTS}

We are grateful for interesting discussions with J. Dawson,

J. DeGroot, J. Denavit, K. Estabrook, C. Joshi, B. Lasinski,

D. Meeker and R.Ziolkowski.

\section{REFERENCES}

1. B. Godrey, W. Shanahan and L. Thode, Phys. Fluids 18, 346 (1975); L. Thode, Phys Fluids 19, 305 and 316 (i976); and many references therein.

2. K.G. Kato, G. Benford and D. Tzach, Phys. Fluids 26, 3636 (1983); and references therein.

3. A. B. Langdon and B. F. Lasinski, in Methods in Computational Physics, edited by J. Killeen, B. Alder, S. Fernbach, and M. Rotenberg, Vol. 16, p. 327 (Academic, New York, 1976).

4. C. Joshi, W. Mori, T. Katsouleas, J. Dawson, J. Kindel and D. Forslund, Nature 311, 525 (1984); D. W. Forslund, J. M. Kindel, W. B. Mori, C. Joshi and J. M. Dawson, Phys. Rev. Letters 54, 558 (1985); and references therein.

5. C. Joshi, private communication.

6. J. Dawson, Private communication. 